\title{
Methodology for constructing an environmental barometer based on artificial intelligence technologies
}

\author{
Vladimir Glinskiy ${ }^{1,2, *}$, Lyudmila Serga ${ }^{2}$, and Mikhail Alekseev ${ }^{2}$ \\ ${ }^{1}$ Novosibirsk State University of Economics and Management, 630102, Novosibirsk, Russia \\ ${ }^{2}$ The Russian Presidential Academy of National Economy and Public Administration - the Siberian \\ Institute of Management, 630102, Novosibirsk, Russian Federation
}

\begin{abstract}
The presented article is aimed at solving the problem of implementing effective quality management of the regional ecosystem and preventing environmental threats. Trends in the reduction of species biological diversity and climate change are observed. Forecast and prevention of large-scale emergencies of various nature should become the basis for a new environmental and economic policy, at the center of which should be an updated system of environmental regulation. The problem arises of standardizing the quality of the environment, which requires the development of a methodology for its assessment based on a certain tool that determines the permissible or unacceptable anthropogenic load and thereby warns of the emergence of a threat. The scientific novelty of the research consists in the development of a methodology for constructing an environmental monitoring system (IAS "Environmental Barometer"), aimed at measuring negative pressure on the territorial ecosystem and based on a digital information and analytical system used by federal and regional authorities for effective management of environmental quality and sustainable development of the territory on the basis of formed strategies. The scientific novelty of the research consists in the development of a methodology for the implementation of environmental monitoring.
\end{abstract}

\section{Introduction}

Over the past decades, foci of ecological trouble have periodically formed in Russia, which negatively affects the quality of life of people, their health and life expectancy. Trends in the reduction of species biological diversity and climate change are observed. The increase in the negative impact of man and production on the environment is also evidenced by official statistics. About 15 percent of Russia's territory is in a critical or near-critical condition in terms of environmental indicators. The declared economic growth may lead to a further exacerbation of environmental problems if the current level of negative impact remains and the failure to take measures to reduce the accumulated environmental damage. Forecast and prevention of large-scale emergencies of various nature should become the basis for a new

* Corresponding author: glinskiy-vv@ ranepa.ru 
environmental and economic policy, at the center of which should be an updated system of environmental regulation.

The goal of environmental policy is to maintain the quality of the natural environment and the environmental conditions of human life at least at the current level, to form a balanced environmentally oriented model for the development of the economy and environmentally competitive industries. The successful implementation by Russia of the environmental development program is associated with the establishment of standards for the permissible anthropogenic load for the territories, the prevention of environmental threats, the typology of territories according to the degree of environmental hazard (the level of concentration of its pollution, which poses a threat to the health and life of the population living on them) and the determination of quantitative and qualitative benchmarks for the development of local environmental programs and regulation of the negative impact of economic entities. Thus, the problem arises of standardizing the quality of the environment, which requires the development of a methodology for its assessment based on a certain tool that determines the permissible or unacceptable anthropogenic load and thereby warns of the emergence of a threat. This tool, in turn, will make it possible to typologize territories and determine a strategy for their development in order to prevent an environmental catastrophe or maintain a comfortable living environment.

\section{Research methodology}

The scientific novelty of the research consists in the development of a methodology for constructing an environmental monitoring system (IAS "Environmental Barometer"), aimed at measuring negative pressure on the territorial ecosystem and based on a digital information and analytical system used by federal and regional authorities for effective management of environmental quality and sustainable development of the territory on the basis of formed strategies. Such a system opens up wide opportunities for preventing natural and man-made threats to the regional economic system in order to maintain its sustainable economic growth and environmental and social stability.

The following results are expected: theoretical foundations of building the IAS "Environmental Barometer" for the development of territorial units through the integrated use of official data and Big Data and assessing its effectiveness. Research is focused on the development of an approach to use the "Environmental Barometer" not so much in the plane of highlighting weak, medium and high environmental hazards, but through the establishment of its boundaries, leading to a regional catastrophe, and management of economic development and environmental and social stability of the region through the mechanism monitoring and control over the level of environmental hazard based on the application of the developed frame management strategies. The design of the research is notable for its novelty, and the results obtained contribute to the development of the theory of sustainable development in terms of the mechanism for preventing environmental threats and managing the ecology of regional economic systems.

The target group of the project is government bodies, the Ministry of Natural Resources and Environment of the Russian Federation, local government bodies, an academic audience.

The theoretical significance of the research lies in the development of the theory and methodology of environmental statistics in terms of improving statistical monitoring of the level of environmental safety of territories, based on the integrated use of official statistics, Open Data and Big Data.

The practical significance of the work lies in the possibility of using the research results and recommendations, first of all, by the executive authorities in the process of managing the territory and ensuring its sustainable development based on economic growth, environmental safety and social stability, secondly, by the services of the Ministry of Regional Development 
and the Ministry of Natural Resources and Ecology of the Russian Federation and individual subjects in monitoring the state of the ecological situation, identifying "problem" areas and preventing environmental threats.

The scientific novelty of the research consists in the development of a methodology for the implementation of environmental monitoring, based on a digital information and analytical system, including an assessment of the level of environmental hazard, a typology of territories according to the degree of existing danger and the receipt of warning signals about an environmental threat, and used by federal and regional authorities for effective quality management environment and sustainable development of the territory on the basis of formed strategies.

\section{Results of the study}

\subsection{A brief research history for constructing «Environmental Barometer»}

Attention of the scientists in various fields of study in the world is focused on the matters of research of ecological situation, determination of the level and problems of ecological safety, assessment of ecological risk and formation of ecological policy. Ecological safety and ecological risks are considered through the prism of both specific applied research conducted by ecologists, biologists, chemists and representatives of engineering scientific thought, and through assessment and modeling of social and economic, political and demographical consequences of negative and dangerous human and natural impact on the environment. The current state of science favors the view that it's impossible to research a complex object of the study within just one field of science, it's necessary to consider it within a system approach as a meta-subject quasi-object of the study and use vast tools of many fields of science: ecology, economics, sociology, statistics, informatics and many others. Besides, considering the fact that the study is devoted to construction of the environmental barometer of certain territories, the observed objects should be considered from the perspective of spatial analysis. For this reason, analysis of the modern state of the studies regarding the matters of ecological monitoring can be carried out along four key theoretical dimensions: studies of ecological safety and risks; spatial analysis; cognitive research of social and economic systems and predictive analytics based on theory of social media.

I. Regarding the first dimension - studies of ecological safety and risks, it should be noted that numerous researchers in the world are involved in this problematic.

Many governmental international organizations deal with the matters of global ecological safety, in particular, there are several continuous programs related to the United Nations, such as: UNEP — United Nations Environmental Program; MAB - a continuous program "Man and the Biosphere Programme" within UNESCO activities; programs of assessment of transboundary pollutants transport and study of the impact on ozone layer of the Earth within the World Meteorological Office activities. Besides, the World Health Organization's international subprograms of health protection, programs of lean and sustainable production by International Labor Organization and International Atomic Energy Agency are directly linked to environmental protection. International non-governmental organizations like World Bank, World Wildlife Fund, International Union for the Protection of Nature, Greenpeace and others are extensively involved in their scientific research and activities regarding conservation of natural objects and resources. Ecological safety has become the central object of studies of leading Russian scientific organizations including those within the Russian Academy of Sciences a long time ago. For instance, the Center of Ecological Safety of the Russian Academy of Sciences is involved in research of ecological safety and assessment of ecological risks of the territories; the main vector of research includes the methods of early 
ecologic and chemical diagnosis and prevention of ecological safety threats, economical and legal issues of provision of ecological safety in the territory of the Russian Federation. Apart from the Center of Ecological Safety of the Russian Academy of Sciences, scientists of the V.B. Sochava Institute of Geography SB RAS deal with the matters of ecological hazard and risk. Besides, there are numerous studies of certain aspects of ecological safety.

II. Many domestic and international studies are devoted to spatial approach and certain matters of the theory and methodology of spatial analysis.

The longstanding existing concept of spatial analysis as a component of the location theory is being replaced with a broader understanding - as a methodology of studying natural and economic systems in terms of justification of their formation, distribution and transformation in space. These studies are based on economic and mathematical models of location of production, population, interregional economic relations. Besides, spatial analysis is based on unification of the theory and methodology of spatial economy, economic geography and regional science in the position of territorial paradigm [1]. The specifics of regional science, theory of regional analysis and location theory are now considered as the components of spatial economy within spatial and dynamic studies.

III. The following can be noted regarding modern experience in conducting cognitive study of fuzzy spatial objects which include clusters, agglomerations, metropolitan cities and adjacent territorial concentration. Russian and foreign scientists attempted to summarize the concept of cognitive economy on the basis of inclusion of some sections of intellectual systems in economy, models, based on knowledge, soft computing and cognitive business analytics into this field and reveal the prerequisites for emergence of this direction in modern economic theories.

Russian and foreign scientists were involved in the matters of structural analysis of complex fuzzy structured systems. Unstable events and processes on the basis of the total concept of data typology, the methods of assessment of parameters of object types with fuzzy boundaries, determination of optimal criteria of threshold and fuzzy structured spatial objects are researched, in particular, by V.V. Glinskiy [2 - 5], L.K. Serga [2 - 5], M.A. Alekseev [2, 3, 6] and K.A. Zaykov [4, 5].

IV. In modern societies, social economy and ecology relationships are now almost inseparable from technology use, to the extent that interactions through social media platforms. A vast majority of our communication takes place not just through physical encounters, but mainly through technological platform mediation. According to G. Lovink the term " "platform' was strategically chosen to present the contradictory activities of online services as a neutral ground for...users and major media producers, while enabling the collision of privacy and surveillance efforts, community and advertising investments" [7]. P. Markano said, that "taking our current intellectual and material capabilities into consideration, we must explore the extent to which certain needs perpetuate toil, which are characteristic of their falsity, and the extent to which their satisfaction could lead to a more liberated society (ecologically organized society), which are characteristic of their truth" [8].

Social media platform analysis has been extensively used to study natural resources governance and management $[9,10]$. This is so because the management of natural resources typically involves a large variety of actors, who are embedded in various institutions and interact at different scales and in heterogeneous ways [11].

The named phenomena are indicative of wider kinds of social, economic and ecological change with which new media are associated: 1) a shift from modernity to postmodernity; 2) intensifying processes of globalization and overuse of environment; 3) a decentering of established and centralized geopolitical orders and praxis [12]. However, a number of studies and bodies of thought that attempt to address the nature of everyday life and experience in advanced technological societies under the name of 'cyberculture' and/or 'cybercultural studies' do have some things to say about interactions between economy, technology and 
environment [7, p. 318]. A. Anderson places the media politics of the environment protection within the wider context of debates concerning the role of contemporary media in communicating of ecological and economical risks [13], and mentioned, that in recent years a number of scholars have highlighted the lack of sustained, rigorous analysis of the role of the media and media platforms in reporting risks concerning with human activity.

Thus, the problems of construction of the ecological monitoring system on the base of technological platform «Environmental Barometer» as a tool for measurement of pressure of production activity on ecology, assessment of the level of ecological hazard in the region, receiving warning signals from federal and regional authorities regarding ecological threat to effective management of sustainable development of the territory were not considered in scientific literature.

\subsection{Core methodology for constructing an «Environmental Barometer»}

So, the theory of sustainable development, theory of rational use of natural resources, ecological safety, total concept of data typology and theory of fuzzy sets, prediction theory and decision-making theory form the theoretical basis for constructing an environmental barometer.

The methodological base of the study is the authorial scientific approach based on summary of actual practice of regional management in the conditions of market economy; methods confirming fundamental principles of functioning of the territorial economic systems in the context of ecological safety, such as: wholeness (change of the system element leads to system change in general), integrity (system can have properties which are not found in its elements and vice versa), hierarchy (system is an element of the system of higher level), synergism (overall result of the system development is bigger than sum of the results of development of its elements); typologies and models of management of social and ecological and economic development of the territorial entities; methods of decision making on the basis of the theory of sustainable development.

The assumption that system adaptability has a "natural" limit is important for the development of the robust control of economic - ecological systems. The limit in the management theory serves as information transformer into system behavior. Studying the role of the limit in management was originated by the principle of cybernetics and feedback control model.

According to the evolution laws, adaptation is carried out within some limit where actions are considered as threshold response to changes. The assertion that system adaptation is carried out via some shift in the trajectory of its functioning without violation of the threshold values of the limit set by external and internal environment is essential in determination of the mechanisms of adaptive management. Such system behavior conforms to the evolution principle - "punctuated equilibrium".

Adaptive decisions within the limit should place the system in a certain trajectory of "floating equilibrium", like some time attractor. This is followed by the notion of "homeostasis" - a special information structure, which defines the system capability to maintain sustainable dynamic development and ensures its progressive development in a changeable external and internal environment and "robust limit" - some reasonable "norm" of economic and organizational changes in homeostatic indicators of the activity of social and economic systems from the perspective of meeting goals.

Unpredictable variability with different fluctuation categories and aggressiveness of the system environment generated a need for expansion of the hard limit boundaries for the purpose of creation of the condition for dampening non-random nature of factors through preventive development of the options of transferring the system into a particular equilibrium state. Management in this context is known as robust (strength) control, unifying many 
methods of the management theory aimed at synthesis of such regulator that would ensure maintenance of the output variables of the system within the robust limit for all types of uncertainty.

Let us formulate five principles which will be considered as base knowledge for the development of the theory of flexible adaptation of economic systems via robust control:

- adaptation can be carried out within some limit, restricted by thresholds of possible actions and stability;

- adaptive actions are considered as threshold response to changes;

- adaptation goal - to transform the system state through the established mechanism of management of the "ensemble of significant parameters", presented by variables at the entry of the system, in order to reach the values that provide matching of the output performance indicators of the system functioning and values set by the robust limit;

- adaptive decisions within the limit place the system in a certain trajectory of "floating equilibrium", i.e. a certain time attractor;

- limits of adaptive response can be eliminated under the influence of destabilizing factors, brought in by chaos and uncertainty, mostly carried by polysubjective environment surrounding the system, with changes in the adaptive norm.

Let us establish that the mechanism of robust control is a certain configurator which synthesizes various ideas of strategic, tactical and prompt actions of the management system and makes an elective selection for creation of the "floating equilibrium" in homeostatic space, ensuring robust stability of functioning and development of the social and economic system.

The frame method of knowledge organization should be considered as a method of floating periodization of processing time series of economic data for elimination of the disparity between existing and required knowledge, needed for creation of robust stability of economic systems. The mechanism of robust control is created on the basis of developed cognitivism (formation of expanded context of external environment knowledge) and constructivism (transformation of knowledge into some constructions of frames: models of strategy, tactics and prompt actions). Each element of the mechanism of robust control at the design phase is aimed at negotiation of uncertainty. Thus, the strategy is focused on negotiation of environmental uncertainty. The development of optimization plans is carried out with consideration for the impact, primarily, of object uncertainty, and owing to their correspondence to the strategy, of environmental uncertainty as well. When moving on to implementation of the plans on a per day basis, immersion into situational uncertainty concurrently with negotiation of object uncertainty takes place. [6]

Manuel Castells [14] defines that frames are primarily neural networks of associations which can become available using language in combination with metaphors. A research study by Glinskiy et al. [3-5] showed that neural network organization of frames makes it possible to overcome the lack of available input information and make correct assessment of the possible conditions of economic systems in the future.

\section{Discussion of the results}

At that, framing means activation of specific neuron networks. Alongside with that, it is mentioned in the book by Entman [15] that framing is a process of "picking (selection) and formulation of some aspects of events or problems and establishing connection between them so as to facilitate the spread of certain interpretation, assessment and (or) solution".

Consideration of the provisions of the presented research area forms the basis for development of the system of monitoring and management of social and economic system from the perspective of environmental threat prevention on the basis of construction of the 
"environmental barometer" using artificial intelligence technology, unparalleled anywhere in the world.

Theoretical bases of construction of the "Environmental barometer" IAS of the development of the territorial entities via complex use of official data and Big Data and assessment of its efficiency. The studies are aimed at the development of the approach for the purpose of use of the "Environmental barometer" not so much in marking out low, medium or high environmental hazard but in determination of its boundaries, leading to a regional catastrophe, and management of economic development and ecological and social stability of the region via the mechanism of monitoring and control of the level of ecological hazard on the basis of use of the developed frame management strategies. Statement of the research is notable for novelty, and obtained results contribute to the development of the theory of sustainable development relating to the mechanism of environmental threats prevention and management of ecology of regional economic systems.

\section{Conclusion}

Methods of adequate assessment of the level of ecological hazard to social and economic development of the territory, relevant to all levels of spatial hierarchy; the results of application of the methods form the methods of determination of the boundary, leading to environmental threat and imbalance of the parameters of the state of territorial social and economic system. Contrary to generally accepted understanding of the boundary as a critical point of transition from one state to another, the project related research is aimed at marking out types of territorial systems with various level of ecological instability from the perspective of destimulation of economic growth. A system of frame management strategies for threat prevention or maintaining stability is established for each type of territories.

A frame model (in the "actions menu" format) of management of the territory in the context of elaboration and application of normative ecological strategies depending on its ecological type from the perspective of the state and dynamics of the process. Such a model opens multiple possibilities for prevention of natural and technogenic threats to regional economic system for the purpose of maintaining its sustainable economic growth and ecological and social stability.

The reported study was funded by Russian Foundation for Basic Research according to research projects No 20-010-00560.

\section{References}

1. S.V. Makar, Vestnik of Financial University, 2 (68), 61 (2018)

2. M.A. Alekseev, V.V. Glinskiy, L.K. Serga, M.L. Pyatov, M.L., Voprosy Statistiki, 26, 7, 20 (2019)

3. V.V. Glinskiy, L.K. Serga, M.A. Alekseev, Procedia Manuf., 43, 263 (2020)

4. V. Glinskiy, L. Serga, M. Khvan, K. Zaykov, Procedia Manuf., 21, 494 (2017)

5. V. Glinskiy, L. Serga, M. Khvan, K. Zaykov, Procedia Manuf., 8, 315 (2017)

6. S.E. Khrushchev, M.A. Alekseev, O.M. Logachova, Voprosy Statistiki, 26 (2), 27 (2019)

7. G. Lovink, Social Media Abyss: Critical Internet Cultures and the Force of Negation (Cambridge, UK: Polity, 2016)

8. P.E.B. Marcano, Honors Theses (PPE), 35 (2018), https://repository.upenn.edu/ppe_honors/35 
9. Ö. Bodin, C. Prell, Social networks and natural resource management. Uncovering the social fabric of environmental governance (Cambridge University Press, Cambridge, UK, 2011)

10. B. Crona, K. Hubacek, Ecology and Society Special Feature, 48 (2010

11. M. Salpeteur, I. Díaz-Reviriego, L. Calvet-Mir, V. Reyes-García, Ecology and Society, 22 (2017)

12. M. Lister, J. Dovey, S. Giddings, K. Kelly, New Media: a critical introduction (Taylor \& Francis e-Library, 2008)

13. A. Anderson, Media, Environment and the Network Society (Palgrave Macmillan, UK, 2014)

14. M. Castells, Communication Power (Oxford University Press, Oxford (GB), 2009)

15. R.M. Entman, Projections of Power: Framing News, Public Opinion, and US Foreign Policy (University of Chicago Press, Chicago (IL), 2004) 